\title{
Feasibility of biventricular volume and function assessment using first-pass gated ${ }^{15} \mathrm{O}$-water PET
}

\author{
Fayçal Ben Bouallègue ${ }^{1,2,3^{*}}$ D, Denis Mariano-Goulart ${ }^{1,2}$, Denis Agostini ${ }^{3}$ and Alain Manrique ${ }^{3,4}$
}

\begin{abstract}
Background: We investigated the feasibility of left ventricular (LV) and right ventricular (RV) volume and function estimation using a first-pass gated ${ }^{15} \mathrm{O}$-water PET. This prospective study included 19 patients addressed for myocardial perfusion reserve assessment using ${ }^{15} \mathrm{O}$-water PET. PET data were acquired at rest and after regadenoson stress, and gated first-pass images were reconstructed over the time range corresponding to tracer first-pass through the cardiac cavities and post-processed using TomPool software; LV and RV were segmented using a semiautomated 4D immersion algorithm. LV volumes were computed using a count-based model and a fixed threshold at $30 \%$ of the maximal activity. RV volumes were computed using a geometrical model and an adjustable threshold that was set so as to fit LV and RV stroke volumes. Ejection curves were fitted using a deformable reference curve model. LV results were compared to those obtained using ${ }^{99 \mathrm{~m}} \mathrm{Tc}$-sestamibi gated myocardial SPECT in terms of end-diastolic volume (EDV), end-systolic volume (ESV), stroke volume (SV), and ejection fraction (EF).

Results: There was an excellent concordance between rest and stress PET in terms of EDV and ESV (Lin's coefficient $0.85-0.90)$, SV $(\sim 0.80)$, and EF $(\sim 0.75)$ for both ventricles. Correlation with myocardial SPECT was high for LV EDV (Pearson's $R=0.89, p<0.001)$ and ESV $(R=0.87, p<0.001)$ and satisfying for $\operatorname{LV} S V(R=0.67, p<0.001)$ and $\mathrm{EF}(R=0.67, p<0.001)$. Minimal LV ESV overestimation $(+4 \mathrm{~mL}, p=0.03)$ and $\mathrm{EF}$ underestimation $(-4 \%, p=0$. 01) were observed using PET.
\end{abstract}

Conclusions: Biventricular volume and function assessment are achievable using the first-pass PET, and LV parameters correlate well with those derived from gated myocardial SPECT.

Keywords: ${ }^{15} \mathrm{O}$-water PET, First-pass, Ventricular volume, Ventricular function

\section{Background}

Left ventricle (LV) function assessment is of utmost importance in the initial evaluation and during follow-up of patients with LV systolic [1] or diastolic [2] dysfunction or receiving cardiotoxic chemotherapy [3]. Imaging right ventricle (RV) function, albeit technically more challenging and less routinely implemented, may provide relevant diagnostic or prognostic information in patients with heart failure [4], after inferior myocardial infarction [5], or in more specific disorders such as amyloidosis [6] or

\footnotetext{
* Correspondence: faycal.ben-bouallegue@umontpellier.fr

${ }^{1}$ Nuclear Medicine Department, Montpellier University Hospital, Montpellier, France

${ }^{2}$ PhyMedExp, INSERM - CNRS, Montpellier University, Montpellier, France Full list of author information is available at the end of the article
}

arrhythmogenic dysplasia [7]. Standard LV or RV function assessment requires the estimation of end-diastole (ED) and end-systole (ES) volumes as well as ejection fraction $(\mathrm{EF})$, which can be achieved using various non-invasive imaging techniques including echocardiography, cardiac magnetic resonance (CMR), and isotopic techniques. In the frame of nuclear medicine, cardiac function is usually studied by means of gated equilibrium radionuclide angiography, using either planar techniques or single photon emission computerized tomography (SPECT) and ${ }^{99 \mathrm{~m}} \mathrm{Tc}-$ labelled red blood cells (RBC) [8-10]. LV function analysis using ${ }^{15} \mathrm{O}-\mathrm{CO}$ blood-pool positron emission tomography (PET) has been proven to be achievable [11]. LV functional parameters may alternatively be inferred from perfusion images obtained using dedicated tracers such as 
${ }^{99 m}$ Tc-sestamibi (for SPECT) [12], ${ }^{82} \mathrm{Rb}$ [13] or ${ }^{13} \mathrm{~N}$-ammonia [14] (for PET), or viability images obtained using ${ }^{18}$ FDG PET [15]. Most of these isotopic techniques have been thoroughly validated against $C M R$ as the reference standard $[8,9,11,14-16]$.

Planar first-pass radionuclide angiography using ${ }^{99 \mathrm{~m}}$ Tc-labelled agents has been extensively employed for decades for LV and RV function analysis [17]. The excellent count sensitivity of PET technology makes it an ideal means for dynamic tomographic imaging of fast transient phenomena such as radiotracer transit through cardiac cavities. The steadily increasing use of PET (and particularly cardiac PET) in clinical routine reinforces the motivation to develop first-pass PET methods for cardiac function evaluation. The feasibility of cardiac output and stroke volume (SV) estimations at first-pass by means of the Stewart-Hamilton indicator-dilution principle has already been demonstrated using either ${ }^{82} \mathrm{Rb}$ [18], ${ }^{18}$ FDG [19], or ${ }^{15} \mathrm{O}$-water PET [20], the provided estimates being in close agreement with those of RBC-SPECT, echocardiography, and CMR. Todica et al. described LV volume and function measurements in healthy rats using first-pass gated ${ }^{18} \mathrm{FDG}$ PET [21], without significant difference compared to CMR in terms of ejection fraction. Such cardiac function assessment using first-pass gated PET has been poorly investigated in humans since only two studies are reported on the feasibility of LV function assessment using first-pass gated ${ }^{15} \mathrm{O}$-water PET $[22,23]$.

TomPool is a free software developed in Montpellier University Hospital and designed for semi-automated post-processing of equilibrium blood-pool SPECT images. It has previously been validated against CMR for LV and RV function assessment [8]. It relies on a watershed immersion algorithm for LV and RV segmentation and a deformable reference model for time-activity curve fitting $[24,25]$.

The aim of the present work was to investigate the feasibility of LV and RV volume and function measurement using the first-pass ${ }^{15} \mathrm{O}$-water PET in a cohort of patients referred for myocardial perfusion assessment at rest and after vasodilator stress. First-pass PET was validated against gated myocardial SPECT for LV measurements.

\section{Methods}

\section{Patients}

Nineteen patients aged 47-75 years (median 66 years, 14 men and 5 women) were prospectively recruited from the outpatients of the Nuclear Medicine Department at Caen University Hospital from December 2015 to November 2016 in the frame of the WATERDAY study (ClinicalTrials.gov unique identifier NCT 02278497) aiming to compare myocardial perfusion and flow reserve using dynamic myocardial SPECT to
${ }^{15} \mathrm{O}$-water PET and endovascular fractional flow reserve measurement [26]. Inclusion criteria were the presence of at least one significant ( $\geq 50 \%)$, non-occlusive, coronary stenosis on percutaneous coronary angiography, and no history of myocardial infarction. All patients underwent both myocardial SPECT and ${ }^{15} \mathrm{O}$-water PET within 3-17 days (median 10 days), without any relevant cardiovascular event or change in cardiotropic medication between the two examinations. The study was approved by the Regional Ethics Committee (CPP Nord-Ouest III, France), and all patients gave written informed consent. The clinical characteristics of the study population are summarized in Table 1.

\section{Myocardial SPECT acquisition and analysis}

All SPECT acquisitions were carried out using a dedicated CZT cardiac SPECT camera (D-SPECT; SpectrumDynamics, Biosensors, Caesarea, Israel) with the patient in supine position. Rest and stress acquisitions were performed in the same session. Rest images were acquired during $5 \mathrm{~min}$, 6-7 $\mathrm{min}$ after injection of $2.5 \mathrm{MBq} / \mathrm{kg}$ of ${ }^{99 \mathrm{~m}}$ Tc-sestamibi. For stress imaging, $7 \mathrm{MBq} / \mathrm{kg}$ of ${ }^{99} \mathrm{~m}$ Tc-sestamibi was injected at peak hyperaemia following IV injection of $400 \mu \mathrm{g}$ of regadenoson, and data were acquired 6-7 min later over $2 \mathrm{~min}$. Gated SPECT images were reconstructed using the manufacturer's dedicated software and post-processed using commercially available software (Corridor4DM, INVIA, Ann Arbor, MI) [27] to obtain LV end-diastolic volume, end-systolic volume, stroke volume, and ejection fraction. The $R-R$ interval was divided into 16 intervals. Myocardial wall

Table 1 Characteristics of the study population

\begin{tabular}{ll}
\hline Male gender & $14(74 \%)$ \\
Age (years) & $65 \pm 8[47-75]$ \\
CCS angor class 1 & $13(68 \%)$ \\
CCS angor class 2 & $6(32 \%)$ \\
Cardiovascular risk factors & \\
BMI > 30 kg/m ${ }^{2}$ & $3(16 \%)$ \\
Diabetes mellitus & $5(26 \%)$ \\
Hypertension & $11(58 \%)$ \\
$>3$ cardiovascular risk factors & $5(26 \%)$ \\
Medical therapy & \\
Antiplatelet & $17(89 \%)$ \\
Beta-blocker & $8(42 \%)$ \\
ACE inhibitor or AT-ll antagonist & $10(53 \%)$ \\
Calcium channel blocker & $3(16 \%)$ \\
Long acting nitrates & $2(11 \%)$ \\
\hline
\end{tabular}

Cardiovascular risk factors include obesity, diabetes mellitus, hypertension, dyslipidaemia, smoking, and family history

CCS Canadian Cardiovascular Society, BMI body mass index 
segmentation was fully automated, and additional slight manual adjustment of LV basal limit was performed whenever necessary.

\section{${ }^{15} \mathrm{O}$-water PET acquisition and analysis}

All PET acquisitions were achieved using a GE Discovery VCT RX scanner (GE Healthcare, Buc, France) and started with a low-dose transmission CT scan for attenuation correction. Two intravenous injections of ${ }^{15} \mathrm{O}$-water $(1.5$ to $3 \mathrm{MBq} / \mathrm{kg}$ ) were performed, each one being simultaneously performed with the start of a dynamic gated 4'30" emission scan. The second emission scan was performed at peak vasodilator stress following IV injection of $400 \mu \mathrm{g}$ regadenoson. After correction for random coincidences, scatter, and dead time, the acquired list-mode data was reconstructed twice. First, a dynamic 24 -frame sequence $(14 \times 5$ ", $3 \times$ $10^{\prime \prime}, 3 \times 20^{\prime \prime}$, and $\left.4 \times 30^{\prime \prime}\right)$ was obtained using Fourier rebinning and 2D filtered back-projection. It allowed to extract a time-activity curve (TAC) in a region of interest (ROI) centred on the cardiac area. TACs were characterized by a sharp early peak corresponding to tracer first-pass through the right then left cardiac cavities followed by a sustained decreasing plateau corresponding to myocardial uptake then wash out. End of tracer first-pass was detected by visual inspection as the TAC transition point between the peak and the plateau. A second image reconstruction was then performed over the first-pass time range using 3D OSEM (nine subsets, two iterations) with eight-interval gating. Reconstructed images were re-oriented into cardiac canonical axes using CardIQ software on a dedicated workstation (Advantage Windows 4.4, GE Healthcare, Buc, France). Final image matrices were sampled on a $64 \times 64 \times 47$ grid with $3.27 \mathrm{~mm}$ cubic voxels.

First-pass gated blood-pool images were post-processed using in-house software (TomPool, freely available for download at http://scinti.edu.umontpellier.fr) that was originally designed for equilibrium radionuclide angiography and adapted for first-pass data processing. Initial operator intervention was required to set the position of the septal plane (on horizontal long axis images), the valve plane (on vertical long axis images), and the infundibular plane as the upper limit of the RV (on short-axis images). A four-dimensional (4D) immersion algorithm was then run that produced a partition of the gated images into 4D regions centred on local intensity maxima. Each region was then assigned either to LV, RV, or extra-ventricular activity depending on the relative position of its barycentre with respect to the reference planes. Manual correction for misassigned regions was possible as well as semi-automated segmentation refinement. LV segmentation mask was obtained using a thresholding method at a fixed percentage of the 4D maximal intensity value inside the regions belonging to LV. Thresholds ranging from 20 to $40 \%$ were tested in order to determine the optimal threshold value. LV volumes were computed using a count-based method in which the volume of each voxel was normalized by the ratio of its intensity to the LV maximal intensity. RV segmentation mask was obtained by applying an adjustable threshold with respect to the $4 \mathrm{D}$ maximal intensity value inside the regions belonging to $\mathrm{RV}$, and $\mathrm{RV}$ volumes were computed using a geometric method in which each voxel accounted for a constant volume regardless of its intensity. The RV threshold was chosen by the operator based on visual inspection and so as to fit as much as possible RV and LV stroke volumes (which was legitimate since no patient had documented valvular disease). Ventricular ejection curves were fitted to the eight time samples $\left(\left\{t_{n}, v_{n}\right\}, n=1 \ldots\right.$ 8) using a deformable curve model $v_{n}=\eta\left[R\left(t_{n}\right.\right.$ $\left.+\tau\left(t_{n}\right)\right)$ ] where $R(t)$ is the reference curve derived from a series of normal patients sampled over 512 time points, $\eta$ a second-order polynomial, and $\tau$ a weighted sum of cosine functions with variable phase shift and frequency.

\section{Statistical analysis}

Continuous variables are expressed as mean \pm standard deviation (min-max range). Concordance and correlation between rest and stress functional parameters obtained using first-pass PET were assessed using Pearson's correlation coefficient $(R)$, Lin's concordance correlation coefficient (ccc), and Bland-Altman analysis. Comparison between LV volume and function given by first-pass PET and those given by myocardial SPECT was achieved using the same metrics. Difference between homologous variables was characterized using a paired Student's $t$ test after checking data sample normalcy using the Kolmogorov-Smirnov test. All statistical computations were carried out using Excel (Microsoft, Redmond, WA).

\section{Results}

Injected activities were $399 \pm 88 \mathrm{MBq}(175-521)$ for rest PET, $300 \pm 81 \mathrm{MBq}$ (131-459) for stress PET, $295 \pm$ $57 \mathrm{MBq}(169-419)$ for rest SPECT, and $774 \pm 128 \mathrm{MBq}$ (558-1012) for stress SPECT. Subject haemodynamic parameters are detailed in Table 2. All patients were in sinus rhythm, and no significant cardiac arrhythmia was observed in any SPECT or PET study ( $R-R$ interval rejection $=0 \%$ for all PET acquisitions and $<10 \%$ for all SPECT acquisitions).

Visual analysis of perfusion myocardial SPECT data did not reveal advanced ischaemia (i.e., affecting more than 
Table 2 Subject haemodynamic parameters in PET and SPECT studies

\begin{tabular}{|c|c|c|c|c|}
\hline & \multicolumn{2}{|l|}{ PET } & \multicolumn{2}{|l|}{ SPECT } \\
\hline & Rest & Stress & Rest & Stress \\
\hline Heart rate (bpm) & $69 \pm 13$ & $82 \pm 24^{*}$ & $68 \pm 15$ & $88 \pm 21^{*}$ \\
\hline $\begin{array}{l}\text { Systolic BP } \\
(\mathrm{mmHg})\end{array}$ & $116 \pm 15$ & $118 \pm 20$ & $129 \pm 21^{\neq}$ & $134 \pm 21^{\neq}$ \\
\hline $\begin{array}{l}\text { Diastolic BP } \\
(\mathrm{mmHg})\end{array}$ & $60 \pm 10$ & $59 \pm 14$ & $68 \pm 12^{\ddagger}$ & $73 \pm 12^{\ddagger}$ \\
\hline $\begin{array}{l}\text { Rate-pressure } \\
\text { product }\end{array}$ & $8000 \pm 1540$ & $9810 \pm 3890^{*}$ & $8770 \pm 2380$ & $\begin{array}{l}11,780 \pm \\
3500 * \neq\end{array}$ \\
\hline
\end{tabular}

$B P$ blood pressure

*Significantly different from rest value $(p<0.05)$

FSignificantly different from PET value $(p<0.05)$

two myocardial segments) in any of the 19 patients. An antero-septo-apical scar was evidenced in one patient.

Regarding first-pass PET, tracer bolus transition time through the cardiac cavities assessed on centred TACs was $25 \pm 4 \mathrm{~s}(18-35)$ for rest acquisitions, corresponding to a total of $37 \pm 12(8-58) 10^{6}$ prompt events, and $22 \pm$ $5 \mathrm{~s}$ (13-32) for stress acquisitions, corresponding to a total of $21 \pm 8(5-33) 10^{6}$ prompt events.

Table 3 details the results of first-pass PET data post-processing using TomPool (LV threshold at 30\%) in terms of left and right ventricular end-diastolic volume, end-systolic volume, stroke volume, and ejection fraction. Mean value and range are indicated at rest and pharmacological stress for each parameter. Difference between stress and rest values (mean and its 95\% confidence interval) is given in the third column. No statistically significant bias was observed between rest and stress volumes. SV and EF were minimally higher at stress for both $\mathrm{LV}(\mathrm{SV}+4.5 \mathrm{~mL}, p=0.04$; $\mathrm{EF}+3 \%, p=$ $0.03)$ and RV $(\mathrm{SV}+5.1 \mathrm{~mL}, p<0.01 ; \mathrm{EF}+4 \%, p<0.01)$. Stroke volumes for $\mathrm{LV}$ and RV were respectively $61 \pm$
$13 \mathrm{~mL}$ versus $60 \pm 12 \mathrm{~mL}$ at rest (not significant) and 65 $\pm 16 \mathrm{~mL}$ versus $65 \pm 15 \mathrm{~mL}$ at stress (not significant).

Figures 1 and 2 show the correlation and Bland-Altman plots comparing the volume and function measurements (from left to right: EDV, ESV, SV, and EF) between rest and stress studies for LV and RV, respectively. For both LV and $\mathrm{RV}$, there was an excellent concordance between rest and stress results in terms of ED and ES volume ( $\mathrm{ccc} \geq 0.85$, all $p$ values $<0.001)$ and a substantial concordance in terms of ejection fraction (ccc $=0.73, p<0.001$ for $\mathrm{LV}$; $\mathrm{ccc}=0.77$, $p<0.001$ for RV).

Table 4 details the bias, correlation (Pearson's coefficient), and concordance (Lin's coefficient) between first-pass PET and myocardial SPECT LV functional parameters depending on the LV segmentation threshold (20 to $40 \%$ by steps of $5 \%$ ). Overall, the $30 \%$ threshold appeared to achieve the best concordance with myocardial SPECT, except in terms of EF for which the 35\% threshold performed slightly better.

Figure 3 displays both correlation and agreement between first-pass PET (LV threshold at 30\%) and gated myocardial SPECT for LV volume and ejection fraction estimates. The scatter plot and the Bland-Altman diagram for each parameter (EDV, ESV, SV, and EF) are shown. The correlation was good for EDV and ESV estimations (respectively $R=0.89$ and $0.87, p<0.001$ ) and fair for SV and EF estimations $(R=0.67, p<0.001$ for both). Compared to myocardial SPECT, first-pass PET tended to slightly overestimate EDV $(+1 \mathrm{~mL}$, not significant, limits of agreement $[-28,31 \mathrm{~mL}])$ and ESV $(+4 \mathrm{~mL}, p=0.03$, limits of agreement $[-16,24 \mathrm{~mL}])$, and to moderately underestimate $\mathrm{SV}(-3 \mathrm{~mL}$, not significant, limits of agreement $[-29,24 \mathrm{~mL}])$ and EF ($4 \%, p=0.01$, limits of agreement $[-23 \%, 15 \%])$.

Figure 4 is built up of screen captures from TomPool after post-processing a rest first-pass ${ }^{15} \mathrm{O}$-water

Table 3 First-pass PET estimates of left and right ventricular volume and function at rest and after pharmacological stress. LV was segmented using a 30\% threshold. The bias (difference between stress and rest value) is given along with its $95 \%$ confidence interval

\begin{tabular}{|c|c|c|c|c|c|c|}
\hline & \multicolumn{2}{|l|}{ Rest PET } & \multicolumn{2}{|l|}{ Stress PET } & \multicolumn{2}{|l|}{ Difference } \\
\hline & Mean \pm SD & $\overline{\text { Range }}$ & $\overline{M e a n} \pm$ SD & $\overline{\text { Range }}$ & (Stress-rest) & \\
\hline LV EDV $(m L)$ & $96 \pm 28$ & $60-161$ & $98 \pm 30$ & $57-167$ & +1.9 & {$[-3.3,7.2]$} \\
\hline LV ESV (mL) & $36 \pm 18$ & $12-77$ & $33 \pm 18$ & $9-66$ & -2.5 & {$[-6.3,1.2]$} \\
\hline LV SV (mL) & $61 \pm 13$ & $39-84$ & $65 \pm 16$ & $47-106$ & +4.5 & {$[0.1,8.8]^{*}$} \\
\hline LV EF (\%) & $65 \pm 10$ & $48-83$ & $68 \pm 10$ & $49-89$ & +3.4 & {$[0.4,6.3]^{*}$} \\
\hline RV EDV (mL) & $113 \pm 28$ & $70-175$ & $115 \pm 28$ & $70-180$ & +2.1 & {$[-2.2,6.3]$} \\
\hline RV ESV (mL) & $53 \pm 19$ & $24-82$ & $50 \pm 19$ & $22-82$ & -3.0 & {$[-6.3,0.3]$} \\
\hline RV SV (mL) & $60 \pm 12$ & 43-93 & $65 \pm 15$ & $46-108$ & +5.1 & {$[1.9,8.2]^{* *}$} \\
\hline RV EF (\%) & $54 \pm 8$ & $41-74$ & $58 \pm 9$ & $42-77$ & +3.6 & {$[1.7,5.6]^{* *}$} \\
\hline
\end{tabular}

$L V$ left ventricle, $R V$ right ventricle, EDV end-diastolic volume, ESV end-systolic volume, SV stroke volume, EF ejection fraction ${ }^{*} p<0.05 ;{ }^{* *} p<0.01$ 


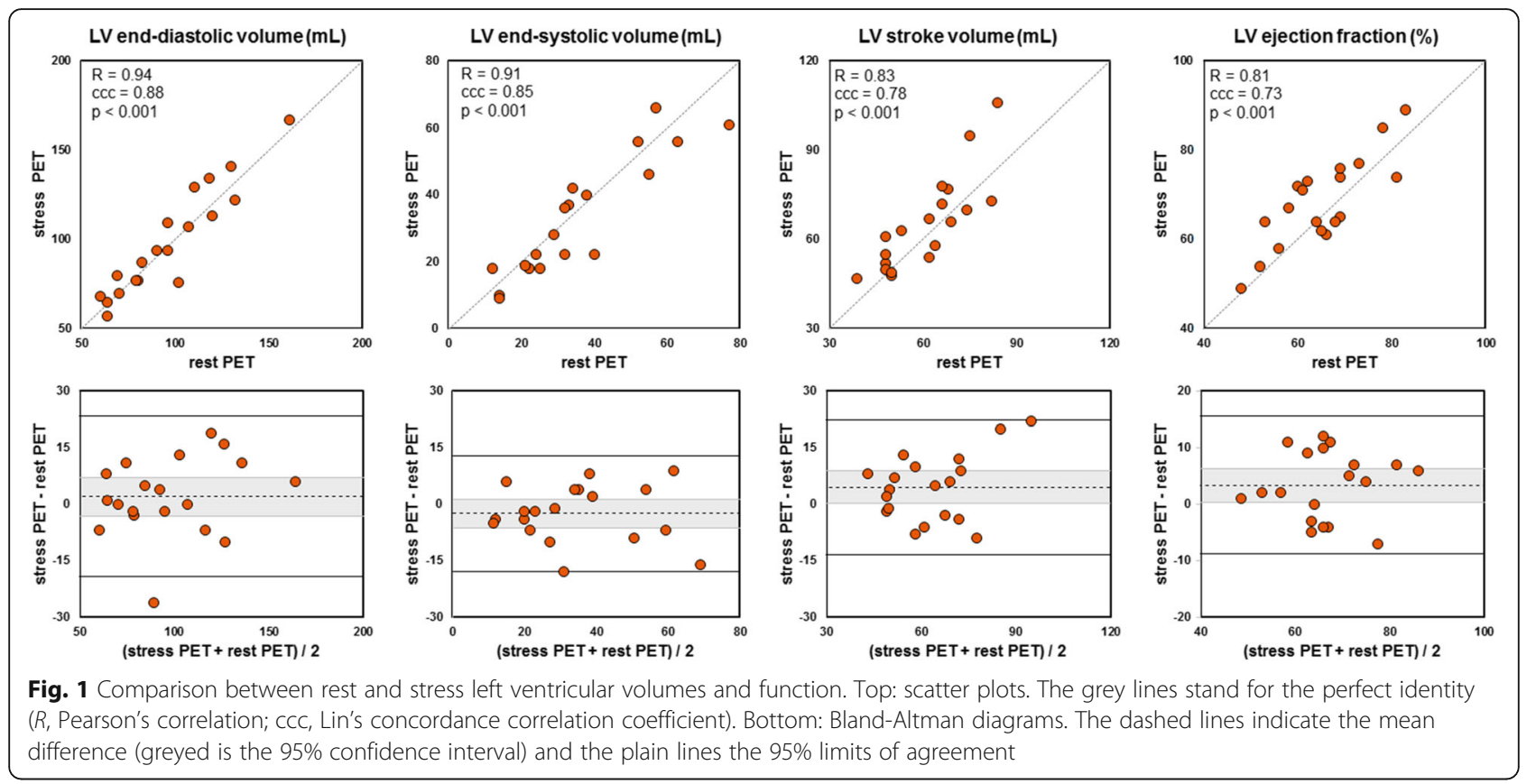

PET study. It displays the horizontal long-axis slices and corresponding segmentation (red: LV, blue: RV, green: extra-ventricular activity) at end-diastole and end-systole, the parametric surfaces built from the segmentation masks (red: LV, blue: RV) at each gating time sample, and the time-volume curves for LV (red) and RV (blue) computed using the deformable curve method.

\section{Discussion}

To our knowledge, this is the first study to investigate the feasibility of biventricular function evaluation using first-pass gated PET in humans. The proposed methodology was implemented in the frame of myocardial perfusion PET using ${ }^{15} \mathrm{O}$-water, but it is readily transposable to any PET examination using any radiopharmaceutical. One would naturally think of the utility


Fig. 2 Comparison between rest and stress right ventricular volumes and function. Top: scatter plots. The grey lines stand for the perfect identity ( $R$, Pearson's correlation; ccc, Lin's concordance correlation coefficient). Bottom: Bland-Altman diagrams. The dashed lines indicate the mean difference (greyed is the $95 \%$ confidence interval) and the plain lines the $95 \%$ limits of agreement 
Table 4 Bias, Prearson's correlation (R), and Lin's concordance (ccc) between first-pass PET and myocardial SPECT LV functional parameters according to the LV segmentation threshold

\begin{tabular}{|c|c|c|c|c|c|c|c|c|c|c|c|c|}
\hline \multirow[t]{2}{*}{ Threshold (\%) } & \multicolumn{3}{|c|}{ LV EDV (mL) } & \multicolumn{3}{|c|}{ LV ESV (mL) } & \multicolumn{3}{|c|}{ LV SV (mL) } & \multicolumn{3}{|c|}{ LV EF (\%) } \\
\hline & Bias & $R$ & $\mathrm{ccc}$ & Bias & $R$ & $\mathrm{ccc}$ & Bias & $R$ & Conc. & Bias & $R$ & $\mathrm{ccc}$ \\
\hline 20 & +12 & 0.89 & 0.83 & +14 & 0.86 & 0.69 & -2 & 0.67 & 0.64 & -10 & 0.66 & 0.45 \\
\hline 25 & +7 & 0.89 & 0.86 & +9 & 0.86 & 0.79 & -2 & 0.67 & 0.65 & -7 & 0.69 & 0.56 \\
\hline 30 & +1 & 0.89 & 0.88 & +4 & 0.87 & 0.85 & -3 & 0.67 & 0.65 & -4 & 0.67 & 0.61 \\
\hline 35 & -5 & 0.89 & 0.87 & -1 & 0.86 & 0.85 & -4 & 0.68 & 0.65 & -2 & 0.66 & 0.64 \\
\hline 40 & -11 & 0.89 & 0.81 & -5 & 0.86 & 0.79 & -6 & 0.67 & 0.61 & +1 & 0.65 & 0.63 \\
\hline
\end{tabular}

$L V$ left ventricle, EDV end-diastolic volume, ESV end-systolic volume, SV stroke volume, EF ejection fraction

of a simultaneous evaluation of the cardiac function in patients referred for myocardial perfusion imaging using ${ }^{82} \mathrm{Rb}$ or ${ }^{13} \mathrm{NH}_{3}$ PET, or for assessment of myocardial viability or cardiac sarcoidosis using ${ }^{18}$ FDG PET. LV function follow-up using ${ }^{18}$ FDG PET would also benefit oncological patients receiving cardiotoxic chemotherapy. Accordingly, in our study, injected activities (1.5 to $3 \mathrm{MBq} / \mathrm{kg}$ ) were adjusted in order to match those usually prescribed in routine ${ }^{18} \mathrm{FDG}$ PET. Due to a longer half-life and shorter positron range ( 0.6 and 1.5 versus $2.5 \mathrm{~mm}$ ) of the isotope, ${ }^{18} \mathrm{FDG}$ and ${ }^{13} \mathrm{NH}_{3}$ images should benefit from higher quality in terms of signal-to-noise ratio and spatial resolution compared to those obtained using ${ }^{15} \mathrm{O}$-water. The main constraint imposed by first-pass imaging was the need for a double reconstruction of the acquired PET data, first in a dynamic mode in order to closely circumscribe the TAC peak then in a gated mode over the time range of the first-pass of
${ }^{15} \mathrm{O}$-water. This two-step method appeared essential for image quality optimization, as attested by preliminary experiments which results are not reported here. Indeed, the blood-pool contrast was clearly diminished when gated images were reconstructed over a fixed time range (30 or $60 \mathrm{~s}$ ) due to a significant myocardial accumulation of ${ }^{15} \mathrm{O}$-water, leading to segmentation inaccuracies and systematic volume overestimations. This issue was critical because of the freely diffusible character of the employed tracer, but it might not be as crucial in the frame of ${ }^{82} \mathrm{Rb}$ and ${ }^{18} \mathrm{FDG}$ PET in which myocardial uptake is expected to be slower compared to tracer first-pass [28].

In-house software was employed for image post-processing owing to its ability to be appropriately upgraded and its versatility of use. The software has been validated against CMR for equilibrium gated blood-pool SPECT using a count-based approach and a
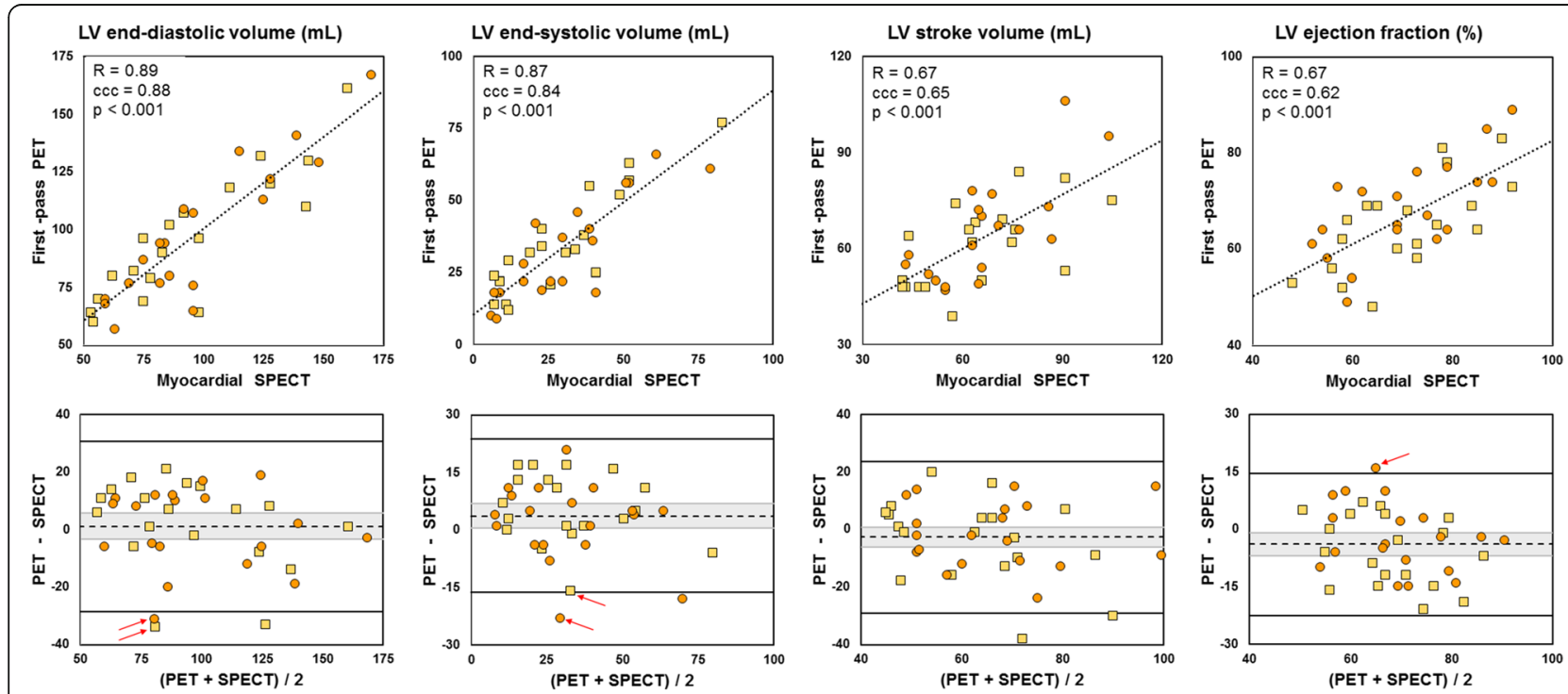

Fig. 3 Correlation and agreement between left ventricular volume and function obtained using myocardial SPECT and first-pass PET. Yellow square markers stand for rest studies and orange round markers for stress studies. Top: scatter plots. The dotted lines stand for the linear regression ( $R$, Pearson's correlation; $c c c$, Lin's concordance correlation coefficient). Bottom: Bland-Altman diagrams. The dashed lines indicate the mean difference (greyed is the $95 \%$ confidence interval) and the plain lines the $95 \%$ limits of agreement. The outliers labelled using red arrows refer to a single patient with an antero-septo-apical scar from prior infarct 




Fig. 4 Example of a rest first-pass ${ }^{15} \mathrm{O}$-water PET study post-processed using TomPool. a Horizontal long-axis slices and corresponding segmentation (red: LV, blue: RV, green: extra-ventricular activity) at end-diastole (top) and end-systole (bottom). $\mathbf{b}$ Parametric surfaces built from the segmentation masks (red: LV, blue: RV) at each gating time sample. c Time-volume curves for LV (red) and RV (blue) computed using the deformable curve model

fixed threshold at $30 \%$ of the maximal intensity [8]. The count-based approach relies on the assumption of a uniform activity inside cardiac ventricles. This method is supposed to account for signal blurring at the ventricle edges due to partial volume effect and motion artefacts. The $30 \%$ threshold was initially chosen for the sake of repeatability and appeared in our study to be that ensuring the closest agreement with gated myocardial SPECT. When passing from equilibrium to first-pass imaging, the uniformity assumption was reasonably maintained for what concerns LV since tracer dilution in blood flow was sufficient downstream the pulmonary circulation [29]. The fixed 30\% threshold was therefore applied with respect to the maximal intensity pixel inside the LV. As regards $\mathrm{RV}$, tracer dilution was not considered effective during tracer transit through right cavities, as witnessed by the signal inhomogeneity near the RV basis at end-systole due to the proximity of a right atrial filling-related "hot spot" (see Fig. 4). To account for this phenomenon, a geometrical model was used along with an adjustable threshold that was tuned by visual inspection and so as to fit as much as possible RV and LV stroke volumes.

The recruited patients were referred for myocardial perfusion PET; hence, rest and stress acquisitions were systematically performed in order to estimate rest and stress absolute blood flow and myocardial flow reserve. Regadenoson stress is known to increase the heart rate by approximately $30 \%$ in healthy subjects [30], without significant changes in blood pressure. A transient decrease in LV EF has been reported in patients with significant ischaemia [31] which was not observed in any of our patients at the time of myocardial SPECT. Therefore, PET rest and stress acquisitions were considered as two replicates of the same examination, allowing for test-retest reliability assessment. In terms of image quality, stress acquisitions were characterized by slightly shorter transit times due to heart rate increase and lower count statistics due to both shorter transit time and lower injected activities. As appreciable from Table 3 and Figs. 1 and 2, rest and stress measurements were highly correlated and concordant. No systematic difference was observed in terms of LV and RV volumes. Minimal EF increase at stress was noted for both LV (+3\%) and RV (+4\%), likely due to a mild adrenergic reaction related to the discomfort following regadenoson injection, and consistent with recently reported results [32]. RV and LV stroke volumes were in close agreement, owing to the employed thresholding method for RV segmentation. Compared to LV, RV volumes were slightly higher and RV EF slightly lower, in line with previously published reference values in healthy subjects $[33,34]$. 
In our study, myocardial perfusion SPECT was used as the reference standard for LV function assessment. This decision was based on a large body of evidence regarding the accuracy and reproducibility of this technique using both Anger [35] and CZT cameras [36, 37]. The choice of the post-processing tool relied on the fact that Corridor4DM yields minimal bias in volume measurements with respect to other commercial software and no significant difference in EF estimations with a comparison to CMR [38]. First-pass PET measurements correlated well with SPECT measurements, especially for LV volumes (Pearson's correlation around 0.9), with similar correlation coefficients for rest and stress studies (results not shown). The low resolution of SPECT images is known to induce ESV underestimation that accounts for the observed difference with PET estimations $(4 \mathrm{~mL})$. As regards EF estimations, first-pass PET resulted in a minimal underestimation $(-4 \%)$ that remained within the reported range of variability of SPECT LV EF estimations [39]. Of note, most of the outliers observed on the Bland-Altman diagrams (5 out of 9, labelled using red arrows in Fig. 3) correspond to a single patient with a significant apical scar from prior infarct, a condition in which gated myocardial SPECT results are subject to caution.

Finally, it has to be noted that stress dynamic PET imaging was started within $1 \mathrm{~min}$ after the completion of regadenoson injection whereas stress gated SPECT was acquired after the completion of dynamic SPECT imaging required for the WATERDAY study, i.e., 6-7 min after regadenoson injection. Theoretically, this may have an impact on LV function assessment using post-stress SPECT imaging. However, Thomas et al. recently demonstrated that the small increase in LV and RV EF observed after regadenoson injection persists during at least $15 \mathrm{~min}$ [32]. Consequently, we believe that this 6-7 min delay would have only a negligible effect on the results of gated SPECT LV function assessment in this study.

\section{Conclusions}

These preliminary results demonstrate that complete LV function assessment is feasible using first-pass gated PET. LV volume and ejection fraction measurements showed satisfying repeatability and are in close agreement with those provided by gated myocardial SPECT. Right ventricular function assessment is also workable, although no reference standard was available for comparison in the present study.

The feasibility of biventricular function assessment using first-pass gated ${ }^{15} \mathrm{O}$-water PET should favour the future development of similar first-pass methods using routine tracers as ${ }^{82} \mathrm{Rb}$. Such methods should benefit from the high resolution and sensitivity of last generation PET scanners and would substantially expand the diagnostic information provided by routine cardiac PET explorations.

\section{Funding}

The WATERDAY study was undertaken at CHU Côte de Nacre, Caen, France, which received funding from Spectrum Dynamics Ltd. The present study was undertaken as part of the FHU REMOD-VHF project.

\section{Availability of data and materials \\ Please contact the author for data requests. \\ Authors' contributions \\ FB participated in the design of the study and data acquisition, performed the statistical analysis, and drafted the manuscript. DA and AM participated in the design of the study and data acquisition. FB and DMG developed the software that was used to post-process PET data. DMG, DA, and AM participated in the data analysis and revised the manuscript. All authors read and approved the final manuscript.}

\section{Ethics approval and consent to participate}

All procedures performed in this study were in accordance with the ethical standards of the institutional and/or national research committee and with the 1964 Helsinki Declaration and its later amendments or comparable ethical standards. Written informed consent was obtained from all individual participants included in the study.

\section{Consent for publication}

Not applicable.

\section{Competing interests}

Pr Denis Agostini is a consultant for Spectrum Dynamics Ltd. There is no other potential conflict of interest relevant to his article.

\section{Publisher's Note}

Springer Nature remains neutral with regard to jurisdictional claims in published maps and institutional affiliations.

\section{Author details}

${ }^{1}$ Nuclear Medicine Department, Montpellier University Hospital, Montpellier, France. ${ }^{2}$ PhyMedExp, INSERM - CNRS, Montpellier University, Montpellier, France. ${ }^{3}$ Nuclear Medicine Department, CHU de Caen, Caen, France. ${ }^{4}$ UNICAEN, EA 4650 SEILIRM, GIP Cyceron, Normandie University, Caen, France.

Received: 20 July 2018 Accepted: 7 September 2018

Published online: 17 September 2018

References

1. Wang TJ, Evans JC, Benjamin EJ, Levy D, LeRoy EC, Vasan RS. Natural history of asymptomatic left ventricular systolic dysfunction in the community. Circulation. 2003;108(8):977-82

2. Flachskampf FA, Biering-Sørensen T, Solomon SD, Duvernoy O, Bjerner T, Smiseth OA. Cardiac imaging to evaluate left ventricular diastolic function. JACC Cardiovasc Imaging. 2015;8(9):1071-93.

3. Murbraech K, Smeland KB, Holte H, Loge JH, Lund MB, Wethal T, et al. Heart failure and asymptomatic left ventricular systolic dysfunction in lymphoma survivors treated with autologous stem-cell transplantation: a national crosssectional study. J Clin Oncol. 2015:33(24):2683-91.

4. Gavazzi A, Berzuini C, Campana C, Inserra C, Ponzetta M, Sebastiani R, et al. Value of right ventricular ejection fraction in predicting short-term prognosis of patients with severe chronic heart failure. J Heart Lung Transplant. 1997;16(7):774-85.

5. Smarz K, Zaborska B, Jaxa-Chamiec T, Tysarowski M, Budaj A. Right ventricular systolic function as a marker of prognosis after ST-elevation inferior myocardial infarction 5-year follow-up. Int J Cardiol. 2016;221:549-53.

6. Bodez D, Ternacle J, Guellich A, Galat A, Lim P, Radu C. Prognostic value of right ventricular systolic function in cardiac amyloidosis. Amyloid. 2016;23(3): 158-67.

7. Mariano-Goulart D, Déchaux L, Rouzet F, Barbotte E, Caderas de Kerleau C, Rossi M, et al. Diagnosis of diffuse and localized arrhythmogenic right 
ventricular dysplasia by gated blood-pool SPECT. J Nucl Med. 2007;48(9): 1416-23.

8. Sibille L, Ben Bouallègue F, Bourdon A, Micheau A, Vernhet-Kovacsik $H$, Mariano-Goulart D. Comparative values of gated blood-pool SPECT and CMR for ejection fraction and volume estimation. Nucl Med Commun. 2011; 32(2):121-8.

9. Nichols K, Van Tosh A, Wang Y, Palestro CJ, Reichek N. Validation of gated blood-pool SPECT regional left ventricular function measurements. J Nucl Med. 2009;50(1):53-60

10. Van Kriekinge SD, Berman DS, Germano G. Assessment of RV function using gated blood pool SPECT. J Nucl Cardiol. 2015;22(3):493-5.

11. Hofman HA, Knaapen P, Boellaard R, Bondarenko O, Götte MJ, van Dockum WG, et al. Measurement of left ventricular volumes and function with O-15labeled carbon monoxide gated positron emission tomography: comparison with magnetic resonance imaging. J Nucl Cardiol. 2005;12(6): 639-44

12. Ather S, Iqbal F, Gulotta J, Aljaroudi W, Heo J, Iskandrian AE, et al. Comparison of three commercially available softwares for measuring left ventricular perfusion and function by gated SPECT myocardial perfusion imaging. J Nucl Cardiol. 2014;21(4):673-81.

13. Chander A, Brenner M, Lautamäki R, Voicu C, Merrill J, Bengel FM. Comparison of measures of left ventricular function from electrocardiographically gated 82Rb PET with contrast-enhanced CT ventriculography: a hybrid PET/CT analysis. J Nucl Med. 2008;49(10):1643-50.

14. Khorsand A, Graf S, Eidherr H, Wadsak W, Kletter K, Sochor H, et al. Gated cardiac 13N-NH3 PET for assessment of left ventricular volumes, mass, and ejection fraction: comparison with electrocardiography-gated 18F-FDG PET. J Nucl Med. 2005;46(12):2009-13.

15. Schaefer WM, Lipke CS, Nowak B, Kaiser HJ, Reinartz P, Buecker A, et al. Validation of QGS and 4D-MSPECT for quantification of left ventricular volumes and ejection fraction from gated 18F-FDG PET: comparison with cardiac MRI. J Nucl Med. 2004:45(1):74-9.

16. Ioannidis JP, Trikalinos TA, Danias PG. Electrocardiogram-gated singlephoton emission computed tomography versus cardiac magnetic resonance imaging for the assessment of left ventricular volumes and ejection fraction: a meta-analysis. J Am Coll Cardiol. 2002;39(12):2059-68.

17. Friedman JD, Berman DS, Borges-Neto S, Hayes SW, Johnson LL, Nichols KJ, et al. First-pass radionuclide angiography. J Nucl Cardiol. 2006;13(6):e42-55.

18. Chen EQ, MacIntyre WJ, Fouad FM, Brunken RC, Go RT, Wong CO, et al. Measurement of cardiac output with first-pass determination during rubidium-82 PET myocardial perfusion imaging. Eur J Nucl Med. 1996;23(8): 993-6.

19. Kreissl MC, Wu HM, Stout DB, Ladno W, Schindler TH, Zhang X, et al. Noninvasive measurement of cardiovascular function in mice with hightemporal-resolution small-animal PET. J Nucl Med. 2006;47(6):974-80.

20. Knaapen $P$, Lubberink M, Rijzewijk $L$, van der Meer RW, Unger M, Germans T, et al. Stroke volume measurements with first-pass dynamic positron emission tomography: comparison with cardiovascular magnetic resonance. J Nucl Cardiol. 2008;15(2):218-24.

21. Todica A, Böning G, Lehner S, Weidl E, Cumming P, Wängler C, et al. Positron emission tomography in the assessment of left ventricular function in healthy rats: a comparison of four imaging methods. J Nucl Cardiol. 2013; 20(2):262-74.

22. Driessen RS, van Timmeren JE, Stuijfzand WJ, Rijnierse MT, Danad I, Raijmakers $\mathrm{PG}$, et al. Measurement of $\mathrm{LV}$ volumes and function using oxygen-15 water-gated PET and comparison with CMR imaging. JACC Cardiovasc Imaging. 2016;9(12):1472-4.

23. Nordström J, Kero T, Harms HJ, Widström C, Flachskampf FA, Sörensen J, et al. Calculation of left ventricular volumes and ejection fraction from dynamic cardiac-gated 150-water PET/CT: 5D-PET. EJNMMI Phys. 2017;4(1):26.

24. Mariano-Goulart D, Collet H, Kotzki PO, Zanca M, Rossi M. Semi-automatic segmentation of gated blood-pool emission tomographic images by watersheds. Application to the determination of right and left ejection fractions. Eur J Nucl Med. 1998;25(9):1300-7.

25. Caderas de Kerleau C, Ahronowitz E, Rossi M, Mariano-Goulart D. Automatic generation of noise-free time-activity curve with gated blood-pool emission tomography using deformation of a reference curve. IEEE Trans Med Imaging. 2004;23(4):485-91.

26. Agostini D, Roule V, Nganoa C, Roth N, Baavour R, Parienti JJ, et al. First validation of myocardial flow reserve assessed by dynamic $99 \mathrm{mTc}$-sestamibi CZT-SPECT camera: head to head comparison with 150-water PET and fractional flow reserve in patients with suspected coronary artery disease. The WATERDAY study. Eur J Nucl Med Mol Imaging. 2018;45:1079-90.

27. Ficaro EP, Lee BC, Kritzman JN, Corbett JR. Corridor4DM: the Michigan method for quantitative nuclear cardiology. J Nucl Cardiol. 2007;14(4):455-65.

28. Choi Y, Brunken RC, Hawkins RA, Huang SC, Buxton DB, Hoh CK, et al. Factors affecting myocardial 2-[F-18]fluoro-2-deoxy-D-glucose uptake in positron emission tomography studies of normal humans. Eur J Nucl Med. 1993;20(4):308-18.

29. Dokoumetzidis A, Macheras P. A model for transport and dispersion in the circulatory system based on the vascular fractal tree. Ann Biomed Eng. 2003; 31(3):284-93.

30. Andrikopoulou E, AlJaroudi WA, Farag A, Lester D, Patel H, Iskandrian AE, et al. The reproducibility and prognostic value of serial measurements of heart rate response to regadenoson during myocardial perfusion imaging. Eur J Nucl Med Mol Imaging. 2016:43(8):1493-502.

31. Brodov $Y$, Fish M, Rubeaux M, Otaki Y, Gransar H, Lemley M, et al. Quantitation of left ventricular ejection fraction reserve from early gated regadenoson stress Tc-99m high-efficiency SPECT. J Nucl Cardiol. 2016;23(6): 1251-61.

32. Thomas DM, Minor MR, Aden JK, Lisanti CJ, Steel KE. Effects of adenosine and regadenoson on hemodynamics measured using cardiovascular magnetic resonance imaging. J Cardiovasc Magn Reson. 2017;19(1):96.

33. Sechtem U, Pflugfelder PW, Gould RG, Cassidy MM, Higgins CB. Measurement of right and left ventricular volumes in healthy individuals with cine MR imaging. Radiology. 1987;163(3):697-702.

34. Pfisterer ME, Battler A, Zaret BL. Range of normal values for left and right ventricular ejection fraction at rest and during exercise assessed by radionuclide angiocardiography. Eur Heart J. 1985;6(8):647-55.

35. Hyun IY, Kwan J, Park KS, Lee WH. Reproducibility of TI-201 and Tc-99m sestamibi gated myocardial perfusion SPECT measurement of myocardial function. J Nucl Cardiol. 2001:8(2):182-7.

36. Cochet H, Bullier E, Gerbaud E, Durieux M, Godbert Y, Lederlin M, et al. Absolute quantification of left ventricular global and regional function at nuclear MPI using ultrafast CZT SPECT: initial validation versus cardiac MR. J Nucl Med. 2013;54(4):556-63.

37. Bailliez A, Lairez O, Merlin C, Piriou N, Valette F, Legallois $D$, et al. Left ventricular function assessment using two different cadmium zinc telluride cameras compared to gamma camera with cardiofocal collimators: dynamic cardiac phantom study and clinical validation. J Nucl Med. 2016;57(9):1370-5.

38. Lipke CS, Kühl HP, Nowak B, Kaiser HJ, Reinartz P, Koch KC, et al. Validation of 4D-MSPECT and QGS for quantification of left ventricular volumes and ejection fraction from gated 99mTc-MIBI SPET: comparison with cardiac magnetic resonance imaging. Eur J Nucl Med Mol Imaging. 2004;31(4):482-90.

39. Kliner D, Wang L, Winger D, Follansbee WP, Soman P. A prospective evaluation of the repeatability of left ventricular ejection fraction measurement by gated SPECT. J Nucl Cardiol. 2015;22(6):1237-43.

\section{Submit your manuscript to a SpringerOpen ${ }^{\circ}$ journal and benefit from:}

- Convenient online submission

- Rigorous peer review

- Open access: articles freely available online

- High visibility within the field

- Retaining the copyright to your article

Submit your next manuscript at $\boldsymbol{\triangleright}$ springeropen.com 